\title{
Estilos de pensamiento en estudiantes universitarios de Buenos Aires ${ }^{1}$
}

\author{
Agustín Freiberg Hoffmann², Fabiana Uriel ${ }^{3}$, Rocío Giselle Fernández \\ Da Lama ${ }^{4}$, Mercedes Fernández Liporace ${ }^{5}$ \\ Universidad de Buenos Aires-Argentina ${ }^{2,3,4,5}$, Consejo Nacional de Investigaciones \\ Cientificas y Técnicas (CONICET) $)^{2,5}$
}

Diferentes situaciones académicas demandan de los alumnos el empleo de estilos de pensamiento específicos para desempeńarse correctamente en el sistema universitario. Esta investigación se propone describir los Estilos de Pensamiento de estudiantes universitarios y su relación con el rendimiento académico. Participaron 225 alumnos universitarios de distintas ramas de estudio. Se ejecutó una prueba MANCOVA que obtuvo efectos multivariados significativos para las variables sexo, rama de estudio y la interacción entre sexo y situación laboral. Luego un análisis correlacional verificó asociaciones estadísticamente significativas entre el rendimiento académico y los estilos en estudiantes de Ciencias Sociales y Humanas. Los resultados se discuten a la luz de la teoría y de implicancias prácticas. Palabras clave: estilos de pensamiento, estudiantes universitarios, rendimiento académico, diferencias individuales.

1 Esta investigación ha sido desarrollada en el marco de un proyecto de investigación financiado por la Universidad de Buenos Aires (20020170100064BA).

2 Doctor en Psicología. Ayudante de primera en la asignatura Teoría y Técnica de Exploración y Diagnóstico Psicológico Módulo I, de la Facultad de Psicología de la Universidad de Buenos Aires. Investigador asistente del CONICET. Dirección postal: General Juan Lavalle 2353 (1052) Capital Federal. Argentina Contacto: afreiberg@psi.uba.ar / agustinfreiberg@gmail. com https://orcid.org/0000-0001-8737-1186

3 Licenciado en Psicología y especialista en Educación. Adjunta en la asignatura Teoría y Técnica de Exploración y Diagnóstico Psicológico Módulo I, de la Facultad de Psicología de la Universidad de Buenos Aires. Dirección postal: General Juan Lavalle 2353 (1052) Capital Federal. Argentina. Contacto: furiel@psi.uba.ar https://orcid.org/0000-0003-1575-9557

4 Licenciado en Psicología. Ayudante de segunda en la asignatura Teoría y Técnica de Exploración y Diagnóstico Psicológico Módulo I, de la Facultad de Psicología de la Universidad de Buenos Aires. Dirección postal: General Juan Lavalle 2353 (1052) Capital Federal. Argentina. Contacto: rociogfernandezdalama@hotmail.com https://orcid.org/0000-0003-1529-2926

5 Doctora en Psicología. Titular en la asignatura Teoría y Técnica de Exploración y Diagnóstico Psicológico Módulo I, de la Facultad de Psicología de la Universidad de Buenos Aires. Investigadora principal del CONICET. Dirección postal: General Juan Lavalle 2353 (1052). Capital Federal. Argentina. Contacto: mliporac@psi.uba.ar. https://orcid. org/0000-0001-7044-8386 


\section{Thinking Styles in College Students from Buenos Aires}

Different academic situations demand the use of specific thinking styles in order to achieve success in higher educational. The study aims to describe college students' thinking styles and to relate them with academic achievement. The sample was composed of 225 college students representing diverse majors. A MANCOVA test revealed significant multivariate effects for sex and major, as well as for the interaction between sex and working status. Correlational analysis verified significant associations between academic achievement and styles for Social and Human Science majors. Results are discussed from theoretical and practical standpoints.

Keywords: thinking styles, college students, academic achievement, individual differences.

\section{Estilos de Pensamento em Estudantes Universitários de Buenos Aires}

Diferentes situaçóes acadêmicas exigem dos alunos o uso de Estilos de Pensamento específicos para um bom desempenho no sistema universitário. Esta pesquisa tem como objetivo descrever os Estilos de Pensamento de estudantes universitários e sua relação com o desempenho acadêmico. 225 estudantes universitários de diferentes ramos de estudo participaram. Foi realizado o teste MANCOVA, que obteve efeitos multivariados significativos para as variáveis sexo, ramo de estudo e interação entre sexo e situação de trabalho. Além disso, uma análise correlacional verificou associaçóes estatisticamente significativas entre desempenho acadêmico e estilos em estudantes de Ciências Sociais e Humanas. Os resultados são discutidos à luz da teoria e implicaçóes práticas.

Palavras-chave: estilos de pensamento, estudantes universitários, desempenho acadêmico, diferenças individuais.

\section{Styles de pensée dans les étudiants universitaires de buenos aires}

Différentes situations académiques exigent des étudiants l'utilisation de styles de pensée spécifiques pour fonctionner correctement dans le système universitaire. Cette recherche vise à décrire les styles de pensée des étudiants universitaires et leur relation avec les performances académiques. Les participants étaient 225 étudiants universitaires de différentes filières. Un test MANCOVA a été réalisé qui a eu des effets multivariés significatifs pour les variables sexe, branche d'étude et interaction entre le sexe et la situation de travail. Ensuite, une analyse corrélationnelle a vérifié les associations statistiquement significatives entre la performance académique et les styles des étudiants en sciences humaines et sociales. Les résultats sont discutés basé sur la théorie et des implications pratiques.

Mots-clés: styles de pensée, étudiants universitaires, performances académiques, différences individuelles. 
Los estudiantes universitarios exhiben actualmente una serie de dificultades que resultan preocupantes. Esta problemática se refleja en distintos indicadores estadísticos. Por ejemplo, si bien entre 2006 y 2015 la cantidad de nuevos inscritos y de graduados se incrementó un $27 \%$ y $47 \%$ respectivamente en Argentina (Ministerio de Educación y Deportes de la Nación Argentina, 2016), tal aumento fue menor que el informado por países vecinos como Brasil y Chile en el mismo período (Centro de Estudios de la Educación Argentina, 2016). Sin embargo, entre 2015 y 2016, únicamente el 30\% de los egresados se graduó en el tiempo establecido por cada facultad (Ministerio de Educación y Deportes de la Nación Argentina, 2016). Tanto la demora en el avance de los estudios como el desgranamiento ocurren principalmente durante el primer año de la carrera (Celma, Adamoli, Gutiérrez \& Santana, 2016; Dillon, 2015; Pintos, 2012), lo cual afecta la permanencia de los alumnos en el sistema universitario. Por ello, suelen analizarse distintos factores que potencialmente intervendrían en los trayectos académicos. Entre ellos se destacan los individuales —características psicológicas y sociodemográficas_- y los ambientales —aspectos sociales, económicos y organizacionales- (García de Fanelli $\&$ Adrogué de Deane, 2015). En este sentido se verifica, por ejemplo, que la elección de carrera se explica en parte por variables como factores económicos, el nivel educativo parental, las actitudes, la creatividad, la autonomía, la capacidad para afrontar desafíos, y la estabilidad laboral entre otras (Hsiao \& Pereira, 2016; Udoh \& Sanni, 2012). Además, se observa que variables tales como la edad, el cambio de estado civil durante la carrera, el obtener o no una beca y el nivel educativo de la madre influyen alternativamente en la deserción o permanencia en el sistema (Bonaldo \& Nobre-Perira, 2016; Casano, Cervero, Nuñez, Almeida \& Bernardo, 2018). Asimismo, la asistencia y participación en clase, la motivación intrínseca, el aprendizaje autónomo y estratégico, 
y la percepción de autoeficacia entre otras, se vinculan con el rendimiento académico (Issahaku, 2017; Renzulli, 2015; Teshome, 2018).

Del conjunto de variables que se analizan habitualmente en estudiantes universitarios, las psicológicas son unas de las que mayor atención han recibido al momento de examinar diferencias individuales y explicar el rendimiento académico (e.g. Kun, Kiss \& Kapitány, 2015; Shahzad, Ahmed \& Ghaffar, 2013; Shekhar \& Devi, 2012; Tulbure, 2012; Zare, 2011). Esto ha impulsado el estudio de distintos constructos, entre los cuales están los Estilos de Pensamiento. Tales estilos describen la preferencia de los individuos para pensar frente a una situación particular. Ese modo habitual de pensamiento se halla determinado por la forma en que el sujeto organiza su cognición (Zhang \& Sternberg, 2006). La presente investigación busca analizar mediante un diseño transversal estas preferencias en estudiantes universitarios de distintas carreras. Se parte así de la hipótesis que plantea la existencia de diferencias en los estilos de pensamiento entre los estudiantes universitarios, y que tales estilos se asocian con el rendimiento académico.

\section{Estilos de pensamiento}

La Teoría del Autogobierno Mental afirma que los distintos tipos de gobierno que existen para organizar las sociedades son reflejo de la mente humana. En esta línea, tanto para gobernar un estado como para que las personas se conduzcan individualmente se vuelve necesario establecer prioridades y distribuir recursos para responder a los cambios que demanda el entorno (Sternberg, 1997). Para ello existen diversas modalidades de organización que se conjugan al momento de encarar distintas situaciones, y que se dividen en Formas, Funciones, Niveles, Alcances e Inclinaciones. Las Formas - Monárquica, Jerárquica, Oligárquica y Anárquica - determinan la manera de abordar las situaciones problemáticas; las Funciones —Ejecutiva, Legislativa y Judicialrefieren al rol que se adopta habitualmente en la resolución de tareas diarias; los Niveles — Global y Local — se vinculan con la planificación de acciones que permiten la resolución de problemas; los Alcances —Externo e Interno - aluden a la preferencia para relacionarse con 
otros; y las Inclinaciones - Conservadora y Liberal — se asocian con el grado de tolerancia hacia los cambios que facilitan la resolución de situaciones problemáticas (Escurra-Mayaute, Delgado-Vásquez \& Quezada-Murillo, 2001; Sternberg, 1997). Como puede advertirse, estas cinco categorías mayores de organización agrupan 13 modos de ordenamiento menor, a los que Sternberg (1997) denomina estilos de pensamiento. De la combinación de estos 13 estilos resulta el modo particular en que cada individuo afronta las distintas situaciones que se le presenten. La tabla 1 resume las características de cada uno de los 13 estilos propuestos por la Teoría del autogobierno mental.

\section{Tabla 1}

Teoría del Autogobierno Mental. Estilos de Pensamiento (Sternberg, 1997)

\begin{tabular}{|c|c|c|}
\hline Organización & Estilo & Descripción \\
\hline \multirow{4}{*}{ Formas } & Monárquica & $\begin{array}{l}\text { Estas personas resuelven un problema a la vez. Se orga- } \\
\text { nizan para cumplir con un objetivo único y dejan de lado } \\
\text { el resto. Intentan resolver los problemas con celeridad, sin } \\
\text { atender todo aquello que se encuentre en su camino y no } \\
\text { les sea útil en ese momento. }\end{array}$ \\
\hline & Jerárquica & $\begin{array}{l}\text { Estos sujetos establecen un orden jerárquico a la hora de } \\
\text { encarar diferentes tareas. Asignan prioridades a metas } \\
\text { deseadas. Son organizados y sistemáticos, y capaces de } \\
\text { afrontar distintas actividades pues saben cómo distribuir } \\
\text { sus recursos, a sabiendas de que deberán resolver el resto } \\
\text { a posteriori. }\end{array}$ \\
\hline & Oligárquica & $\begin{array}{l}\text { Estos individuos son el punto medio entre los monár- } \\
\text { quicos y los jerárquicos. Comparten con los primeros } \\
\text { la preferencia por no establecer orden de prioridades en } \\
\text { las tareas a abordar, y, con los segundos, la intención de } \\
\text { resolver simultáneamente varios problemas. Esto los hace } \\
\text { ser flexibles para reasignar prioridades, lo que los lleva } \\
\text { muchas veces a atender cuestiones recientes e ignorar acti- } \\
\text { vidades anteriores. }\end{array}$ \\
\hline & Anárquica & $\begin{array}{l}\text { Son personas poco sistemáticas, que se interesan por dis- } \\
\text { tintos objetivos. Son poco sociables y suelen desafiar a la } \\
\text { autoridad, por lo que les cuesta apegarse a reglas institu- } \\
\text { cionales. Prefieren resolver los problemas aleatoriamente, } \\
\text { sin seguir un orden en particular. }\end{array}$ \\
\hline
\end{tabular}




\begin{tabular}{|c|c|c|}
\hline Organización & Estilo & Descripción \\
\hline \multirow{3}{*}{ Funciones } & Ejecutiva & $\begin{array}{l}\text { Son sujetos apegados a reglas y normas, y gustan de hacer } \\
\text { aquello que se les pide. Prefieren ambientes estructurados } \\
\text { y valoran realizar las tareas exitosamente según los } \\
\text { lineamientos brindados por otras personas. }\end{array}$ \\
\hline & Legislativa & $\begin{array}{l}\text { Estos individuos se caracterizan por ser creativos. Prefieren } \\
\text { seguir sus reglas y hacer las cosas según sus preferencias. } \\
\text { Eligen situaciones desestructuradas en las que pueden } \\
\text { planificar y crear con libertad. }\end{array}$ \\
\hline & Judicial & $\begin{array}{l}\text { Son personas analíticas, que disfrutan de los proce- } \\
\text { dimientos, las ideas y las normas, entre otras cosas. } \\
\text { Examinan que todo funcione correctamente, dentro de } \\
\text { los parámetros esperables. }\end{array}$ \\
\hline \multirow[t]{2}{*}{ Niveles } & Global & $\begin{array}{l}\text { Se trata de sujetos que se enfocan principalmente en el } \\
\text { todo, en la parte macro de una situación. Suelen ser muy } \\
\text { abstractos ya que se interesan por abarcar los problemas } \\
\text { holísticamente. }\end{array}$ \\
\hline & Local & $\begin{array}{l}\text { Son individuos que se centran en los detalles micro de un } \\
\text { problema, focalizándose en las partes que componen una } \\
\text { situación, por lo que son muy concretos. }\end{array}$ \\
\hline \multirow[b]{2}{*}{ Alcances } & Externo & $\begin{array}{l}\text { Estas personas son sociables, abiertas, que aprecian el tra- } \\
\text { bajo en equipo. }\end{array}$ \\
\hline & Interno & $\begin{array}{l}\text { Son sujetos que se enfocan profundamente en la tarea; son } \\
\text { reflexivos, poco sociables y reservados. Además prefieren } \\
\text { trabajar en soledad. }\end{array}$ \\
\hline \multirow{2}{*}{ Inclinaciones } & Liberal & $\begin{array}{l}\text { Estos individuos gustan de efectuar cambios y de romper } \\
\text { con procedimientos establecidos. Prefieren participar de } \\
\text { situaciones ambiguas e inciertas. }\end{array}$ \\
\hline & Conservador & $\begin{array}{l}\text { Se trata de personas que optan por apegarse a las normas y } \\
\text { procedimientos vigentes. Buscan evitar que las cosas cam- } \\
\text { bien dado que la incertidumbre y ambigüedad no les place. }\end{array}$ \\
\hline
\end{tabular}

Con el propósito de evaluar el modelo propuesto por la Teoría del Autogobierno Mental, se diseña el Inventario de Estilos de Pensamiento o Thinking Syles Inventory — TSI - (Sternberg, 1997). Este se compone de 104 ítems que se responden mediante una escala Likert de 7 opciones. Originalmente el instrumento se construye para indagar los 13 estilos del modelo de partida. No obstante, los trabajos para recabar evidencias de validez realizados en distintos contextos arrojan estructuras factoriales que no responden a aquel (Black \& McCoach, 2008; 
Freiberg-Hoffmann, Uriel, Fernández Da Lama \& Fernández-Liporace, 2019; González-Pienda, et al., 2004; Gutierrez \& Krumm, 2012; León, 2009; Maricuţoiu \& Paloş, 2014; Ochiai, Maie \& Wada, 2016; Yun-Dai \& Feldhusen, 1999). En esta línea se encuentra la versión del instrumento adaptada localmente a población de estudiantes universitarios de Buenos Aires que aísla 7 estilos de pensamiento - Judicial/ Liberal, Ejecutivo/Conservador, Externo, Monárquico, Global, Jerárquico, Legislativo- (Freiberg-Hoffmann, et al., 2019).

Las distintas adaptaciones del Inventario de Estilos de Pensamiento dan cuenta de cierto solapamiento entre los estilos. En atención a este fenómeno Zhang y Sternberg (2005), en base a investigaciones previas y otros modelos teóricos - Biggs, Holland, Torrance, entre otros-deciden, como solución, agrupar los Estilos de Pensamiento en tres Tipos denominados I, II y III. Los estilos reunidos en el Tipo I —Legislativo, Judicial, Global, Jerárquico, Liberal—, se consideran los más adaptativos y remiten a un modo complejo de procesamiento de la información, así como a la preferencia por situaciones poco estructuradas. Esto caracteriza a sujetos holísticos, reflexivos, innovadores, perceptivos, intuitivos, concretos y creativos. El Tipo II agrupa, por su parte, los estilos menos adaptativos -Ejecutivo, Local, Monárquico, Conservador - vinculados con un procesamiento sencillo de la información, que permite un abordaje superficial y poco elaborado de los problemas. Estos individuos se describen como tradicionales, conformistas, sumisos, convencionales, analíticos, juiciosos y secuenciales. Distintas investigaciones verifican que las características personales adaptativas (autoeficacia, creatividad, desarrollo psicológico, motivación) correlacionan positivamente con los estilos del Tipo I y negativamente con los del Tipo II (Cheng, Zhang \& Hu, 2015; Dikici, 2014; Fan \& Zhang, 2009; Zhang, 2010). Finalmente, los estilos reunidos en el Tipo III —Anárquico, Oligárquico, Interno, Externo- se vinculan con preferencias tales como capacidad de emprendimiento, sociabilidad, introversión, y extroversión. Estos estilos complementan a los derivados de los Tipos I y II ya que agregan características psicosociales a los modos de abordar una situación, y varían, por esta razón, 
entre un mayor o menor nivel de adaptación (Zhang \& Sternberg, 2005). Dado que los estilos no son excluyentes sino que pueden combinarse de múltiples modos, es que pueden obtenerse perfiles diversos que dan lugar a distintas preferencias para organizar el pensamiento.

Los estilos de pensamiento - evaluados a través de distintos instrumentos - han sido estudiados en ámbitos muy variados tales como el clínico, carcelario, laboral, y publicitario entre otros (Brinker, Chin \& Wilkinson, 2014; Cheek \& Norem, 2016; Groza, Locander \& Howlett, 2016; Lechner \& Paul, 2017; Mills, Grant, Lechner \& Judah, 2014; Nikčević, Marino, Caselli \& Spada, 2017; Wang, Wang, Keller \& Li, 2016; Wilson et al., 2014; Zeigler-Hill, Mandracchia, Dahlen, Shango \& Vrabel, 2017). Particular énfasis han puesto los especialistas en psicología de la educación en el análisis de esta variable, de cara al logro de una mejora de los aprendizajes estudiantiles en los distintos niveles educativos - primario, medio, universitario- (Bayrami \& Tabatabaei, 2015; Emamipour \& Esfandabad, 2013; Holmes, Liden \& Shin, 2013; Tung \& DeSa, 2010; Yu \& Chen, 2012). También se analizaron los estilos en relación con otras variables que participan en el proceso de aprendizaje — afrontamiento del estrés académico, discapacidades, elección de carrera, estilos parentales, autoeficacia, resiliencia y creatividad, entre otras - (e.g. Cheng, Hu \& Sin, 2016; Fan, 2016; Fan \& Zhang, 2014; Piaw, 2014; Sagone \& De Caroli, 2013; Yuan, Zhang \& Fu, 2017). Los resultados informados han incrementado el conocimiento sobre el concepto, lo cual ha sido útil a la hora de planificar acciones tendientes a la mejora de los procesos de aprendizaje. Por todo esto es que resulta de interés analizar los Estilos de Pensamiento en población local de estudiantes universitarios.

\section{Estilos de pensamiento en estudiantes universitarios}

Distintos estudios describen el modo en que los Estilos de Pensamiento difieren según variables sociodemográficas y académicas. Así, por ejemplo, las mujeres muestran mayor preferencia por el estilo Ejecutivo, mientras que en los varones prevalece el Judicial. Los estudiantes de Humanidades manifiestan una mayor frecuencia del estilo 
Ejecutivo, mientas que en los de Ingeniería predomina el Legislativo (Negahi, Nouri \& Khoram, 2015). También se informan diferencias según género en los estilos Legislativo, Judicial y Ejecutivo. Los primeros dos favorables a los varones y el tercero, a las mujeres. Además, se registra preferencia por el estilo Legislativo en estudiantes de Artes, Ejecutivo en los de Finanzas, Externo para los de Ciencias de la Educación y Conservador para los de Ingeniería (Turki, 2012). Por otro lado, al examinarse diferencias en los estilos de pensamiento según año en curso y nacionalidad de los estudiantes no se advierten resultados significativos (Al-Thani, Al-Thani \& Semmar, 2014). Tampoco se registran asociaciones entre los estilos de pensamiento y la edad (Dikici, 2014). Por último, se verifican asociaciones entre el rendimiento académico y los estilos Legislativo, Ejecutivo, Oligárquico, Monárquico, Anárquico, Jerárquico y Judicial (Fatemi \& Heidarie, 2016).

También se considera que los estilos de pensamiento pueden variar en función de las tareas a desarrollar así como de las circunstancias vitales. Además, pueden ser más o menos apreciados en una época o circunstancia que en otras. Cabe resaltar que los estilos de pensamiento no son positivos o negativos per se, sino más o menos adecuados para cada contexto específico (Sternberg, 1997).

El ámbito educativo local habitualmente favorece algunos estilos y desalienta otros (Aljojo, 2017). Por esta razón, es que resulta de interés estudiar los estilos en el ámbito universitario, a fin de conocer las preferencias del alumnado en sus modalidades de pensamiento, así como su posibilidad de volverlos recursos útiles a la hora de aprender en cada especialidad y caso particular. Elaborar una propuesta de aprendizaje que fomente el éxito de la mayoría de los estudiantes se presenta como un desafío para la enseñanza (Sternberg, 1997). Se propone así, por un lado 1) analizar diferencias en los Estilos de Pensamiento según variables sociodemográficas — sexo, edad, situación laboral— y la variable académica rama de estudio, y por otro, 2) examinar, para cada rama de estudio, la relación entre el rendimiento académico y los Estilos de Pensamiento. 


\section{Método}

\section{Participantes}

Los datos fueron recogidos mediante un muestreo por conveniencia. Participaron 225 estudiantes universitarios de Buenos Aires ( $44.4 \%$ mujeres; $55.6 \%$ varones) de entre 18 y 36 ańos $\left(M_{\text {edad }}=23.74\right.$; $D E=4.05)$. Estos alumnos procedían de una multiplicidad de carreras que fueron agrupadas según rama de estudio de acuerdo con la clasificación propuesta por el Ministerio de Educación y Deportes de la Nación Argentina (2016) (29.3\% Ciencias Sociales, 26.2\% Ciencias Aplicadas, 25.8\% Ciencias Básicas, 18.7\% Ciencias Humanas).

\section{Medición}

La información sociodemográfica y de datos académicos se recogió mediante una encuesta que consultó a los evaluados sobre su sexo, edad, situación laboral, facultad, carrera, año de inicio de los estudios universitarios y número total de asignaturas aprobadas. Las dos últimas variables fueron empleadas para estimar una medida del rendimiento académico entendido en sentido amplio, a través del cociente entre el total de asignaturas aprobadas y la antigüedad del alumno en la carrera, para obtener un índice que correlaciona positivamente con el desempeño estudiantil (De Miguel \& Arias, 1999; Tejedor-Tejedor, 2003).

Los estilos de pensamiento se midieron mediante la versión localmente adaptada del Inventario de Estilos de Pensamiento (Freiberg-Hoffmann, et al., 2019). El instrumento evalúa siete Estilos de Pensamiento -Judicial/Liberal, Ejecutivo/Conservador, Externo, Monárquico, Global, Jerárquico, Legislativo-, a través de 45 ítems que deben responderse según una escala Likert de 5 posiciones que van de Nunca a Siempre. El Judicial/Liberal corresponde a sujetos analíticos interesados en los distintos modos de encarar situaciones problemáticas, que gustan de evaluar ideas y procedimientos típicos y, a la vez, de diseñar nuevas formas para hacer las cosas; el Ejecutivo/Conservador describe a personas metódicas que siguen pautas preestablecidas para 
resolver problemas y que prefieren situaciones estructuradas, con metas y planes fijados con antelación; el tipo Externo caracteriza a individuos sociables, abiertos, que aprecian el trabajo en equipo; el Monárquico, a personas que resuelven un problema a la vez y siguen un objetivo único, dejando de lado el resto; el Global pertenece a sujetos con elevada capacidad de abstracción, enfocados principalmente en abarcar la totalidad de un problema; el Jerárquico incluye a individuos que establecen prioridades en cuanto a las metas y tareas, son organizados, sistemáticos y hacen un buen empleo de sus recursos; el Legislativo, por último, agrupa a personas creativas que prefieren manejarse según sus reglas y preferencias. Esta escala cuenta con evidencias de validez de constructo - análisis factorial exploratorio y confirmatorio- y de consistencia interna, que obtuvieron índices alfas ordinales superiores a .70 en todas la dimensiones.

\section{Procedimiento}

Los datos fueron recogidos durante el horario habitual de clases por un psicólogo entrenado y se contó con los respectivos avales institucionales y de los docentes a cargo de los cursos. Además los evaluados fueron informados sobre el propósito de la investigación y el carácter voluntario y anónimo de su colaboración. Se destacó que la participación podía interrumpirse en el momento en que el participante lo deseara. Se firmó un consentimiento informado y no se ofreció ningún tipo de contraprestación.

\section{Análisis de Datos}

Los datos se analizaron mediante el software SPSS 21. Se aplicó un análisis multivariado de la covarianza (MANCOVA), en el que se tomaron los Estilos de Pensamiento como variables dependientes; las variables sexo, situación laboral y rama de estudios, como factores; y la edad, como covariable.

Para el análisis de la correlación de los Estilos de Pensamiento con el rendimiento académico se segmentó, en primer lugar, la muestra 
según rama de estudio. Ello produjo submuestras con $n$ bajos $(n<70)$, por lo que se vio afectado el supuesto de normalidad de la variable rendimiento académico. Con el fin de superar este inconveniente y de mejorar la simetría de la distribución de los datos, se decidió estimar el logaritmo natural de la variable rendimiento académico (Osborne, 2002).

\section{Resultados}

Se analizó el cumplimiento de los supuestos de homogeneidad y multicolinealidad antes de ejecutar la prueba MANCOVA. Se verificó en primer término la homogeneidad de covarianzas del modelo ( $\mathrm{M}$ de Box $=637.709 ; p>.05$ ), (Huberty \& Petroskey, 2000), como también de las varianzas de cada variable dependiente $(p>.05)$. El cumplimiento de este supuesto habilita la lectura del estadístico Traza de Pillai para la interpretación de los resultados (Finch \& French, 2013). Luego se analizó el supuesto de multicolinealidad entre variables dependientes, que resultó verificado $(r<.90)$ (Tabachnick \& Fidell, 2013).

De la aplicación del MANCOVA se obtuvieron como resultados efectos multivariados significativos para las variables sexo $\left(F_{(7,194)}=\right.$ $\left.3.917 ; p=.001 ; \eta^{2}=.124\right)$ y rama de estudio $\left(F_{(21,588)}=2.031 ; p=.004\right.$; $\left.\eta^{2}=.068\right)$. Además, se registró otro efecto significativo que proviene de la interacción entre sexo y situación laboral $\left(F_{(7,194)}=2.282 ; p=.03\right.$; $\left.\eta^{2}=.076\right)$. De la lectura de los valores del estadístico Traza de Pillai se desprende que la variable rama de estudio (.203) es la que realiza el mayor aporte a la explicación del modelo, seguida de la variable sexo (.124), y, en último lugar, la interacción entre sexo y situación laboral (.076). Del análisis de medias según sexo se verificaron diferencias estadísticamente significativas en los estilos Monárquico $(M=17.93$; DE $=3.51 ; M=19.43 ; \mathrm{DE}=4.27 ; p=.011)$ y Jerárquico $(M=10.52 ; \mathrm{DE}$ $=2.38 ;=11.60 ; \mathrm{DE}=2.12 ; p<.001)$, ambas favorables a las mujeres. En cuanto a la variable rama de estudio, se observaron varias diferencias que se detallan en la tabla 2 . 
Estilos de pensamiento en estudiantes universitarios de Buenos Aires / Freiberg et al.

\section{Tabla 2}

Estilos de Pensamiento. Diferencias según ramas de estudio

\begin{tabular}{|c|c|c|c|c|c|}
\hline \multirow{2}{*}{$\begin{array}{l}\text { Rama de } \\
\text { Estudio }\end{array}$} & & \multicolumn{4}{|c|}{ Estilos de Pensamiento } \\
\hline & & $\begin{array}{c}\text { Judicial/ } \\
\text { Liberal }\end{array}$ & $\begin{array}{c}\text { Ejecutivo/ } \\
\text { Conservador }\end{array}$ & Monárquico & Jerárquico \\
\hline \multirow{2}{*}{$\begin{array}{l}\text { Ciencias } \\
\text { Aplicadas }\end{array}$} & $M$ & $42.22^{\mathrm{AB}}$ & $40.72^{\mathrm{A}}$ & $20.06^{\mathrm{A}}$ & $11.95^{\mathrm{A}}$ \\
\hline & $\mathrm{DE}$ & 1.18 & 1 & .61 & .35 \\
\hline \multirow{2}{*}{$\begin{array}{l}\text { Ciencias } \\
\text { Básicas }\end{array}$} & $M$ & $44^{\mathrm{A}}$ & $39.40^{\mathrm{AB}}$ & $18.27^{\mathrm{AB}}$ & $11.23^{\mathrm{AB}}$ \\
\hline & $\mathrm{DE}$ & 1 & .85 & .52 & .29 \\
\hline \multirow{2}{*}{$\begin{array}{l}\text { Ciencias } \\
\text { Humanas }\end{array}$} & $M$ & $42.13^{\mathrm{AB}}$ & $36.08^{\mathrm{B}}$ & $17.35^{\mathrm{B}}$ & $10.62^{\mathrm{AB}}$ \\
\hline & $\mathrm{DE}$ & 1.27 & 1.08 & .66 & .37 \\
\hline \multirow{2}{*}{$\begin{array}{l}\text { Ciencias } \\
\text { Sociales }\end{array}$} & $M$ & $39.33^{\text {B }}$ & $36.94^{\mathrm{B}}$ & $18.79^{\mathrm{AB}}$ & $10.52^{\mathrm{B}}$ \\
\hline & $\mathrm{DE}$ & 1.03 & .87 & .54 & .30 \\
\hline$F$ & & 3.56 & 4.74 & 3.20 & 3.84 \\
\hline$p$ & & .015 & .003 & .024 & .011 \\
\hline$\eta^{2}$ & & .051 & .066 & .046 & .055 \\
\hline
\end{tabular}

Nota: Letras diferentes indican diferencias estadísticamente significativas entre los grupos.

Con respecto a la interacción entre sexo y situación laboral se halló una diferencia significativa en los estilos Judicial/Liberal y Externo (Tabla 3). 


\section{Tabla 3}

Estilos de Pensamiento. Diferencias según interacción entre sexo y situación laboral

\begin{tabular}{|c|c|c|c|c|c|c|c|c|}
\hline & \multicolumn{4}{|c|}{ Judicial/Liberal } & \multicolumn{4}{|c|}{ Externo } \\
\hline & $\begin{array}{c}\text { Mujeres } \\
\text { no } \\
\text { trabajan }\end{array}$ & $\begin{array}{c}\text { Varones } \\
\text { no } \\
\text { trabajan }\end{array}$ & $\begin{array}{l}\text { Mujeres } \\
\text { trabajan }\end{array}$ & $\begin{array}{l}\text { Varones } \\
\text { trabajan }\end{array}$ & $\begin{array}{c}\text { Mujeres } \\
\text { no } \\
\text { trabajan }\end{array}$ & $\begin{array}{c}\text { Varones } \\
\text { no } \\
\text { trabajan }\end{array}$ & $\begin{array}{l}\text { Mujeres } \\
\text { trabajan }\end{array}$ & $\begin{array}{l}\text { Varones } \\
\text { trabajan }\end{array}$ \\
\hline$M$ & 38.97 & 43.96 & 42.85 & 41.89 & 16.67 & 17.38 & 19.38 & 17.42 \\
\hline $\mathrm{DE}$ & 1.26 & 1.25 & 1.07 & .96 & .69 & .68 & .58 & .52 \\
\hline$F$ & 7.04 & & & & 4.75 & & & \\
\hline sl & .752 & & & & .583 & & & \\
\hline$p$ & .009 & & & & .030 & & & \\
\hline$\eta^{2}$ & .034 & & & & .023 & & & \\
\hline
\end{tabular}

Para el estilo Judicial/Liberal se observó que dentro del grupo de mujeres y varones que no trabajan había una menor preferencia por su uso en las mujeres. No obstante al contrastar ambos sexos que sí trabajan se verificó que las mujeres empleaban más que los varones este estilo (Figura 1).

Con relación al estilo Externo, se obtuvo un resultado similar al arrojado por el estilo Judicial/Liberal. Se halló que también en este estilo había una menor preferencia por su uso en las mujeres que no trabajan, en comparación con varones de la misma situación laboral, y que lo opuesto ocurría al comparar ambos sexos con una situación laboral activa (Figura 2). 
Estilos de pensamiento en estudiantes universitarios de Buenos Aires / Freiberg et al.

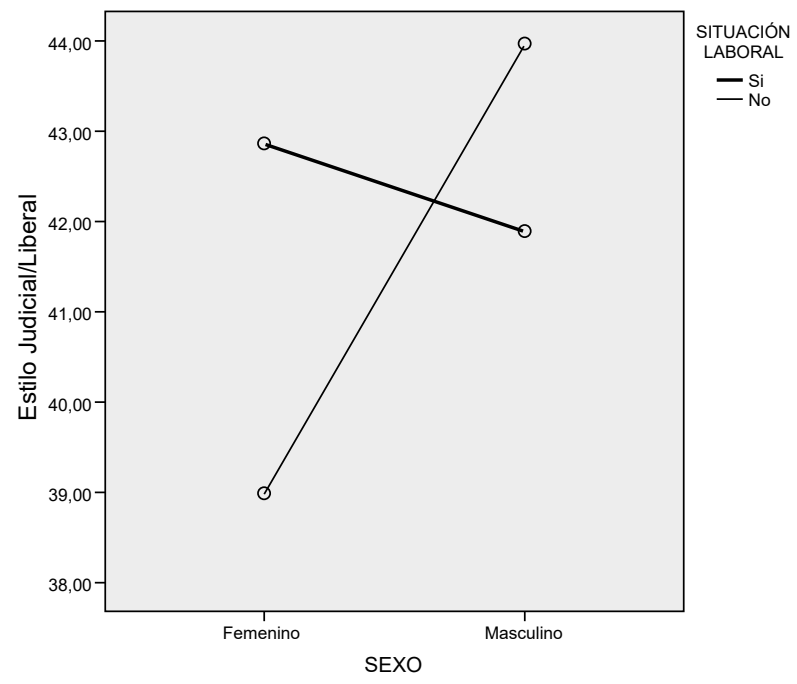

Figura 1. Diferencia del Estilo Judicial/Liberal según sexo y situación laboral.

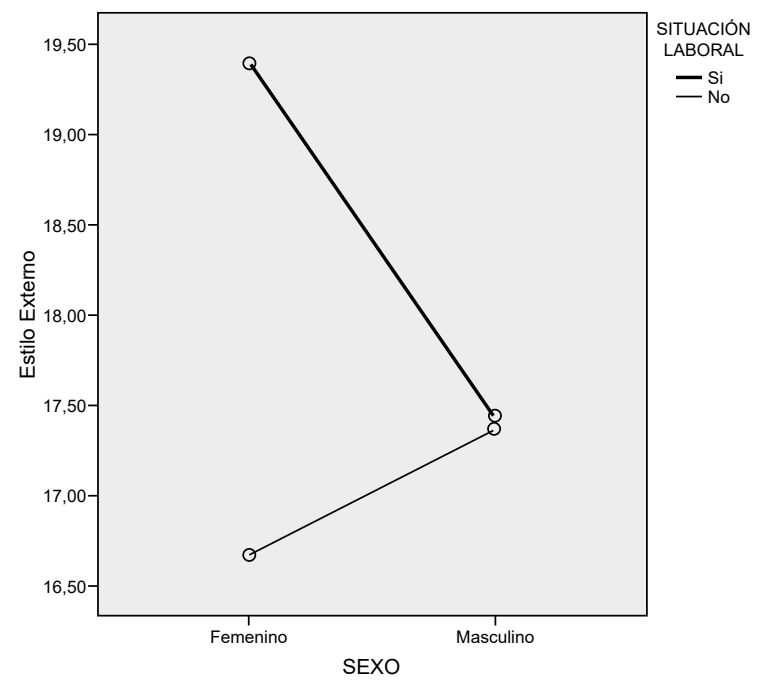

Figura 2. Diferencia del Estilo Externo según sexo y situación laboral. 
Con respecto al segundo objetivo propuesto, se segmentó la muestra según rama de estudio para luego correlacionar la medida del rendimiento académico transformada a logaritmo natural con los Estilos de Pensamiento. De esta manera, se obtuvieron asociaciones estadísticamente significativas entre algunos Estilos de Pensamiento y el rendimiento académico para las ramas de Ciencias Humanas y de Ciencias Sociales (Tabla 4).

\section{Tabla 4}

Correlaciones entre Rendimiento Académico y Estilos de Pensamiento, según rama de estudio.

\begin{tabular}{lcccc}
\hline \multirow{2}{*}{$\begin{array}{l}\text { Rama de } \\
\text { Estudio }\end{array}$} & $\begin{array}{c}\text { Ejecutivo/ } \\
\text { Conservador }\end{array}$ & Monárquico & Jerárquico & Legislativo \\
\cline { 2 - 5 } Ciencias & $-.317^{*}$ & $-.339^{*}$ & $-.428^{* *}$ & $-.378^{*}$ \\
$\begin{array}{l}\text { Humanas } \\
\text { Ciencias } \\
\text { Sociales }\end{array}$ & $-.279^{*}$ & $-.286^{*}$ & $-.258^{*}$ & \\
\hline
\end{tabular}

Nota: ${ }^{*} p<.05 ;{ }^{* *} p<.01$

\section{Discusión}

La presente investigación se propuso examinar el modo en que los Estilos de Pensamiento se presentan en los estudiantes universitarios y cómo estos estilos se relacionan con su rendimiento académico. Con respecto a la variable sexo se obtuvieron diferencias en estilos relacionados con las Formas (Jerárquico y Monárquico), y no con las Funciones (Ejecutiva, Legislativa, Judicial) tal como registraron estudios previos (Negahi, Nouri \& Khoram, 2015; Turki, 2012). Se observó así que las mujeres, a diferencia de los varones, si bien pueden establecer un orden jerárquico, asignar prioridades y recursos a la hora de abordar las tareas académicas (estilo Jerárquico), tienen también una fuerte predilección por resolver un problema a la vez al enfocarse en cumplir con 
ese objetivo específico (estilo Monárquico) y no con varios de modo simultáneo. Una metodología de enseñanza compatible con los Estilos de Pensamiento femeninos debiera centrarse en el trabajo del docente a partir del material de lectura, de modo tal que permita a las estudiantes confeccionar mapas mentales, cuadros sinópticos y toda clase de estrategias que les posibiliten organizar las ideas para luego abordarlas de a una y en orden. Asimismo la metodología de evaluación óptima sería el portafolio, que requiere de parte del alumnado un fuerte compromiso y capacidad para organizarse y autorregularse (Sternberg, 1997).

El análisis según rama de estudio halló distintas preferencias en los Estilos de Pensamiento. En primer lugar se observa que los alumnos de las Ciencias Básicas se destacan por ser analíticos, interesados en los distintos modos de encarar las situaciones problemáticas, así como por su gusto para evaluar ideas y procedimientos típicos, y diseñar nuevas formas para hacer las cosas (Judicial/Liberal). Para este grupo el aprendizaje puede optimizarse si los docentes planifican sus clases para trabajar en grupos pequeños que favorezcan la discusión y el intercambio de ideas. Otra estrategia útil consiste en guiarlos hacia las respuestas correctas mediante el planteo de preguntas que obliguen a emplear un razonamiento hipotético-deductivo; incluso esta misma metodología podría utilizarse en situaciones de evaluación. Otra valoración de los contenidos que se ajuste a este estilo debiera incluir actividades vinculadas a la capacidad analítica (macro y/o microanálisis). El formato puede ser de exámenes sobre temas a desarrollar, o bien multiple choice (Sternberg, 1997; Sternberg \& Williams, 2009). Asimismo pueden contemplarse instancias interactivas con feedback durante la evaluación.

Los estudiantes de las Ciencias Aplicadas se distinguen por su actitud metódica y apegada a pautas preestablecidas para resolver problemas y también por su preferencia por trabajar con metas claras y un plan fijado de antemano (Ejecutivo/Conservador). Además, jerarquizan las actividades a resolver, distribuyen apropiadamente sus recursos (Jerárquico) y son persistentes y tenaces a la hora de responder con un objetivo específico (Monárquico). La metodología de enseñanza que más facilitaría sus aprendizajes es la clase expositiva, que se basa en que el alumno perciba 
la información del modo en que debe ser aprendida, seleccionando aquella porción de datos que le resulte útil según las metas planteadas. Según Sternberg y Zhang (2010) una forma apropiada de evaluar sus aprendizajes es el examen escrito que implique un buen uso de la memoria, organización, y apego a los contenidos dados por los profesores.

En oposición al grupo anterior, los alumnos de Ciencias Humanas y Sociales tienen en común la baja preferencia por los estilos Ejecutivo/ Conservador y Monárquico. Se trata de individuos poco metódicos y persistentes a la hora de resolver problemas; afirman no gustar de plantearse metas y de planificar el modo de alcanzarlas. Esto lleva a sugerir a los profesores evitar metodologías de enseñanza centradas exclusivamente en la transmisión de conocimientos de modo semejante a cómo deben ser aprendidos. Según esta lógica, la utilización de evaluaciones escritas que demanden la puesta en juego de aptitudes tales como la memoria y la organización de los contenidos también podría resultar desventajosa (Sternberg, 1997).

Los resultado arrojados por el análisis MANCOVA sobre la interacción de las variables sexo y situación laboral encontraron diferencias significativas para los estilos Judicial/Liberal y Externo. Se halló, en términos generales, que las mujeres que no trabajan exhiben una preferencia notablemente menor por ambos estilos al compararlas con las mujeres que sí trabajaban y con los varones en general, tanto los que trabajaban como los que no. De esta manera se podría hipotetizar, que, habrían determinados atributos que serían estimulados en las mujeres por el ámbito laboral. Algunos de ellos serían, la capacidad para adoptar un enfoque analítico al momento de encarar las situaciones problemáticas, la evaluación de ideas y procedimientos típicos, y el diseño de formas novedosas para hacer las cosas (Judicial/Liberal) así como también la sociabilidad, la apertura y el trabajo en equipo (Externo). Según se afirma, la formación extra-académica que pueden brindar la familia o bien el ámbito laboral sería capaz de influir en las competencias individuales (Planas-Coll \& Enciso-Avila, 2014; Sternberg, 1997). Con base en esto pueden planificarse acciones tendientes a estimular la preferencia por los estilos Judicial/Liberal y Externo en las mujeres ya 
desde el nivel medio de educación, mediante la organización de pasantías, microemprendimientos, proyectos focalizados o bien a través de estrategias educativas que contemplen la resolución de problemas, el trabajo en equipo y el análisis de situaciones.

Respecto del segundo objetivo que planteaba analizar la relación entre el rendimiento académico y los Estilos de Pensamiento según rama de estudio, se encontraron correlaciones estadísticamente significativas y negativas entre los estilos Ejecutivo/Conservador, Monárquico y Jerárquico tanto para los estudiantes de Ciencias Sociales como para los de Ciencias Humanas. Además se registró otra correlación significativa y negativa con el estilo Legislativo pero solamente para el grupo de Ciencias Humanas. Las asociaciones negativas de estos estilos con el rendimiento académico podrían explicarse para cada estilo de diferente modo. Los estilos Ejecutivo/Conservador y Monárquico corresponden al grupo etiquetado como Tipo II (Zhang \& Sternberg, 2005) que, como se ha mencionado en el apartado teórico, se vinculan con la sencillez cognitiva, el abordaje superficial del conocimiento y su escasa elaboración. Estos estilos son propios de personas tradicionales, conformistas, sumisas a la autoridad, convencionales, analíticas, juiciosas y secuenciales. Si se considera que el sistema universitario prioriza la autonomía de los estudiantes, quienes son libres de administrar el tiempo a su modo, que deben elaborar la información y emplear mayores niveles de abstracción para lograr una apropiada articulación conceptual, resulta lógico que los estilos del Tipo II correlacionen negativamente con el rendimiento estudiantil (Espinoza-Freire, Serrano-Polo \& BritoParedes, 2017). Otras investigaciones han hallado resultados similares para las correlaciones de los estilos de Tipo II y el desempeño académico (e.g. Castro-Solano \& Casullo, 2002; Stanley, Bansh \& Om, 2011). Respecto del estilo Jerárquico, debe decirse que el resultado aquí obtenido se mueve en dirección opuesta a lo teorizado (Sternberg, 1997) y al informado por estudios previos (e.g. Bernardo, Zhang \& Callueng, 2002; Zhang, 2004). En este punto, y dada la data de los trabajos antes citados, sería factible suponer que existan variables intervinientes actuales que podrían modular la relación de este estilo con el rendimiento. 
Por caso, puede pensarse para la muestra analizada en la variable generacional, dado que el 98\% de los estudiantes que la componen corresponde a la denominada Generación Y (Strauss \& Howe 1991). Estos jóvenes se destacan por el manejo de las nuevas tecnologías y además son cooperativos, optimistas, sociables e innovadores. Asimismo, al igual que los de la generación siguiente - Generación Z-, son inquietos y curiosos, cualidades que los llevan a intentar resolver multiplicidad tareas al mismo tiempo - multitasking- (Davies \& Denecker, 2011; Solans \& Lichtmann, 2016). Como puede apreciarse, estas características no están alineadas con las descriptas para el perfil Jerárquico. En este punto podría hipotetizarse que los estudiantes universitarios de hoy, lejos de establecer anticipadamente un orden de prioridades para luego cumplirlo exactamente, intentan resolver las distintas actividades lo más simultáneamente posible, producto ello de su inquietud y capacidad para atender a varios problemas a la vez. Al mismo tiempo, estos alumnos desarrollan paralelamente con su vida académica un mayor número de actividades extracurriculares deportivas, artísticas, culturales, laborales y sociales, entre otras- que contribuyen a su formación integral, de modo tal que sus prioridades no se centran necesariamente en la esfera académica (Guzmán-Brito, 2012; Pozón-López, 2014). Desde este punto de vista podría ser desfavorable para un alumno desatender o postergar algunas tareas en detrimento de otras, puesto que ello podría perjudicar su rendimiento universitario. Queda pendiente para futuros trabajos el análisis de variables posiblemente intervinientes — generacionales, económicas y laborales, entre otras - que puedan ejercer algún efecto mediador entre los Estilos de Pensamiento y el rendimiento estudiantil.

Como último resultado es posible mencionar la relación negativa y significativa entre el estilo Legislativo y el rendimiento académico. Ello se condice con lo reportado en investigaciones previas (e.g. Abaili, 2007; Castro-Solano \& Casullo, 2002; Stanley, Bansh \& Om, 2011). La correlación negativa entre ambas variables se explicaría en que ese estilo se supone incompatible con una buena performance pues es propio de estudiantes creativos que prefieren hacer las cosas de modo diferente, 
y el tipo de enseñanza tradicional predominante penaliza los comportamientos que escapan a las reglas y procedimientos prefijados (Sternberg, 1997). En este sentido, y para seguir las recomendaciones de organismos internaciones (Comisión Económica para América Latina y el Caribe, 2011; Organización de las Naciones Unidas para la Educación, la Ciencia y la Cultura, 2017), sería deseable que los profesores incorporaran metodologías de enseñanza compatibles con la capacidad creativa de los estudiantes, de modo tal de facilitar, por un lado, los aprendizajes y estimular, por otro, la resolución creativa de problemas dado que esta capacidad es cada vez más demandada en el ámbito profesional.

En cuanto a las limitaciones de la investigación, deben destacarse en primer lugar las características de la muestra recogida. Por un lado el tamańo muestral que, si bien resulta aceptable, al segmentarse para realizar comparaciones entre grupos da como resultado $n$ admisibles desde el punto de vista estadístico $(n>30)$ aunque bajos (Ross, 2007). Por otra parte es importante resaltar la dispersión de la muestra en cuanto a carreras y facultades, hecho que obligó a recategorizar esas variables según rama de estudio. Si bien ello responde al agrupamiento de carreras que realiza el Ministerio de Educación y Deportes de la Nación Argentina (2016), sería interesante que futuros trabajos se focalizaran en la recolección de datos en carreras específicas a fin de verificar si la información aquí vertida se replica al interior de cada disciplina académica, para aportar mayor evidencia en favor o en contra de la generalización de estos resultados.

Otra cuestión que debiera considerarse en ulteriores desarrollos es incluir otros instrumentos que evalúen constructos vinculados a los Estilos de Pensamiento, tales como estilos de aprendizaje, estrategias de aprendizaje, enfoques de aprendizaje y rasgos de personalidad, entre otros. Ello posibilitaría complementar la información aquí discutida y valorar interacciones con esas variables o bien efectos moduladores. En esta misma dirección, como ya se ha mencionado, sería interesante recoger también información contextual, ya sea sociodemográfica — nivel socioeconómico autopercibido, nivel educativo parental, ocupación de los padres_, o bien sobre actores que participan 
en la formación del sujeto — estilos de pensamiento docentes, estilos de pensamiento de los agentes de socialización primaria de los alumnos, apoyo social percibido, u otras-, con el propósito de identificar las variables extra-académicas posiblemente relacionadas con los Estilos de Pensamiento.

Una última limitación que merece destacarse corresponde al recorte geográfico de la muestra, que ha sido recogida en universidades ubicadas en el área Metropolitana de Buenos Aires. Aunque esta área concentra el $77.7 \%$ del total de estudiantes de la provincia y de la ciudad de Buenos Aires (Ministerio de Educación de la Nación Argentina, 2013), sería deseable ampliar en el futuro la muestra y así incluir alumnos de la región Bonaerense y otras del país.

Para concluir, este estudio ha tenido por finalidad aportar información para diferentes actores que participan del proceso académico educativo superior. Se espera así que, con base en estos resultados, los docentes reúnan más elementos para adecuar sus prácticas de enseñanza a las preferencias estudiantiles con el fin de facilitar la construcción de conocimientos de manera más particularizada y útil según rama de estudio y sexo. En otro orden, a nivel institucional este informe puede brindar información valiosa para actualizar los planes de estudio, no solamente en cuanto a asignaturas sino al modo de acceso a esos contenidos. Las diferencias de estilos halladas según rama de conocimiento aportan material para considerar que la utilización de algunos de ellos priman por sobre otros según el área. Asimismo, los profesionales psicólogos y psicopedagogos del ámbito educativo también pueden hacer uso de estos resultados para guiar a los alumnos en elecciones disciplinares o de especialidades según sus preferencias de pensamiento. El fin último que se persigue es conseguir mejores aprendizajes y, consecuentemente, evitar demoras y fracasos en los trayectos universitarios que podrían llevar, eventualmente, a desertar del sistema o a cambiar de carrera reiteradamente, con las consecuencias personales, organizacionales y económicas que ello implica.

Futuros trabajos se encargarán de ampliar y complementar lo hasta aquí analizado. 
Estilos de pensamiento en estudiantes universitarios de Buenos Aires / Freiberg et al.

\section{Referencias}

Abaili, M. (2007). Differences in thinking styles among low, average, and high achievement college students. Paper presented at the 13th International Conference on Thinking Norrköping, Sweden. Recuperado de http://citeseerx.ist.psu.edu/viewdoc/download? doi=10.1.1.537.4545\&rep=rep1\&type=pdf

Al-Thani, A., Al-Thani, T. \& Semmar, Y. (2014). Investigation the relationship between students' thinking styles, self-efficacy, for learning, and academic performance at Qatar University. American International Journal of Social Sciences, 3(2), 172-179.

Aljojo, N. (2017). Differences in styles of thinking "in light of Sternberg's theory": A case study of different educational levels in Saudi Arabia. Journal of Technology and Science Education, 7(3), 333-346. https://doi.org/10.3926/jotse.291

Bayrami, M. \& Tabatabaei, S. (2015). Predicate thinking styles based on component of metacognitive styles. Journal of Psychology, 19(1), 71-87.

Bernardo, A, Zhang, L. \& Callueng, C. (2002). Thinking styles and academic achievement among Filipino students. Journal of Genetic Psychology, 163(2), 149-163. https://doi. org/10.1080/00221320209598674

Black, A. \& McCoach, D. (2008). Validity study of the Thinking Styles Inventory. Journal for the Education of the Gifted, 32(2), 180-210. https://doi.org/10.4219/jeg-2008-849

Bonaldo, L. \& Nobre-Perira, L. (2016). Dropout: Demographic profile of Brazilian university students. Procedia - Social and Behavioral Sciences, 228, 138-143. https://doi.org/10.1016/j. sbspro.2016.07.020

Brinker, J., Chin, Z. \& Wilkinson, R. (2014). Ruminative thinking style and the MMPI-2-RF. Personality and Individual Differences, 66, 102-105. https://doi.org/10.1016/j.paid.2014.03.001

Casano, J., Cervero, A., Nuñez, J., Almeida, L. \& Bernardo, A. (2018). Factors that determine the persistence and dropout of university students. Psicothema, 30(4), 408-414. 
Castro-Solano, A. \& Casullo, M. (2002). Predictores del rendimiento académico y militar de cadetes argentinos. Anales de Psicología, 18(2), 247-259.

Celma, G. C., Adamoli, A. M., Gutiérrez, M. C. \& Santana, S. N. (diciembre, 2016). La evolución de las cohortes: Retención, deserción y desgranamiento en la carrera Ingeniería Quimica de la facultad Regional Buenos Aires de la UTN. Recuperado de http:// www.alfaguia.org/alfaguia/files/1320940018_5688.pdf

Centro de Estudios de la Educación Argentina (2016). Ingreso a la universidad en Brasil, Chile y Argentina. Recuperado de http://www. rlcu.org.ar/recursos/E_0000046_010_cea_numero_50.pdf

Comisión Económica para América Latina y el Caribe (2011). Educación, desarrollo y ciudadanía en América Latina. Chile: Cepal.

Cheek, N. \& Norem, J. (2016). Holistic thinkers anchor less: Exploring the roles of self-construal and thinking styles in anchoring susceptibility. Personality and Individual Differences, 115, 174-176. https://doi.org/10.1016/j.paid.2016.01.034

Cheng, S., Hu, X. \& Sin, K. (2016). Thinking styles of university deaf or hard of hearing students and hearing students. Research in Developmental Disabilities, 55, 377-387. https://doi. org/10.1016/j.ridd.2016.04.004

Cheng, S., Zhang, L. \& Hu, X. (2015). Thinking styles and university self-efficacy among deaf, hard-of-hearing, and hearing students. Journal of DeafStudies and DeafEducation, 20(1), 16-26. https:// doi.org/10.1093/deafed/env032

Davies, C. \& Denecker, C. (2011). Meeting them where they are: Millennials, technology, and academic etiquette. International Journal of Applied Science and Technology, 1(5), 68-73.

De Miguel, M. \& Arias, J. (1999). La evaluación del rendimiento inmediato en la enseñanza universitaria. Revista de Educación, 320, 353-377.

Dikici, A. (2014). Relationship between thinking styles and behaviors fostering creativity: An exploratory study for the mediating role of certain demographics traits. Kuram ve Uygulamada 
Eğitim Bilimleri, 14(1), 179-201. https://doi.org/10.12738/ estp.2014.1.1939

Dillon, A. (2015, 5 de marzo). UBA: 1 de cada 5 chicos cambia de carrera en el primer año. Clarín.com, Sociedad, Recuperado de http://www.clarin.com/sociedad/uba-orientacion_vocacional_ 0_H1DVvQ5w7x.html

Emamipour, S. \& Esfandabad, H. (2013). Developmental study of thinking styles in Iranian Students University. ProcediaSocial and Behavioral Sciences, 84, 1736-1739. https://doi.org/ 10.1016/j.sbspro.2013.07.023

Escurra-Mayaute, L., Delgado-Vásquez, A., Quezada-Murillo, R. (2001). Estilos de pensamiento en estudiantes de la U.N.M.S.M. Revista de Investigación en Psicología, 4(1), 9-34. https://doi.org/ 10.15381/rinvp.v4i1.5004

Espinoza-Freire, C., Serrano-Polo, C. \& Brito-Paredes, P. (2017). El trabajo autónomo en estudiantes de la Universidad Técnica de Machala. Universidad y Sociedad, 9(2), 202-212.

Fan, J. (2016). The role of thinking styles in career decision-making self-efficacy among university students. Thinking Skills and Creativity, 20, 63-73. https://doi.org/10.1016/j.tsc.2016.03.001

Fan, J. \& Zhang, L. (2014). The role of perceived parenting styles in thinking styles. Learning and Individual Differences, 32, 204-211. https://doi.org/10.1016/j.lindif.2014.03.004

Fan, W. \& Zhang, L. (2009). Are achievement motivation and thinking styles related? A visit among Chinese university students. Learning and Individual Differences, 19(2), 299-303. https://doi. org/10.1016/j.lindif.2008.10.005

Fatemi, M. \& Heidarie, A. (2016). Relationship between thinking styles and academic achievement of the students. International Journal of Humanities and Cultural Studies, 2(4), 1353-1361.

Finch, H. \& French, B. (2013). A Monte Carlo comparison of robust MANCOVA test statistics. Journal of Modern Applied Statistical Methods, 12(2), 35-81. https://doi.org/10.22237/ jmasm/1383278580 
Freiberg-Hoffmann, A., Uriel, F., Fernández Da Lama, R. \& FernándezLiporace, M. M. (2019). Inventario de Estilos de Pensamiento: Propiedades psicométricas en universitarios de Buenos Aires. Interacciones, 5(2). https://doi.org/10.24016/2019.v5n2.165

García de Fanelli, A. \& Adrogué de Deane, C. (2015). Abandono de los estudios universitarios: Dimensión, factores asociados, y desafíos para la política pública. Revista Fuentes, 16, 85-106. https://doi. org/10.12795/revistafuentes.2015.i16.04

González-Pienda, J., Núńez, J., González-Pumariega, S., Álvarez, L., Roces, C., González, P., ... \& Sales, P. (2004). Estilos de pensamiento: análisis de su validez estructural a través de las respuestas de adolescentes al Thinking Styles Inventory. Psicothema, 16(1), 139-148.

Groza, M., Locander, D. \& Howlett, C. (2016). Linking thinking styles to sales performance: The importance of creativity and subjective knowledge. Journal of Business Research, 69(10), 4185-4193. https://doi.org/10.1016/j.jbusres.2016.03.006

Gutierrez, M. \& Krumm, G. (2012). Adaptación y validación del Inventario de Estilos de Pensamiento de Sternberg (TSI) en la provincia de Entre Ríos-Argentina. Interdisciplinaria, 29(1), 43-62. https://doi.org/10.16888/interd.2012.29.1.3

Guzmán-Brito, J. (2012). Modelos predictivos y explicativos del rendimiento académico universitarios: caso de una institución privada en México (Tesis doctoral). Recuperado de http://eprints.ucm. es/15335/1/T33748.pdf

Holmes, R., Liden, S. \& Shin, L. (2013). Children's thinking styles, play, and academic performance. American Journal of Play, 5(2), 219-238.

Hsiao, J. \& Pereira, S. (2016). Generational approach to factors influencing career choice in Accounting. Revista Contabilidade \& Finanças, 27(72), 393-407. https://doi.org/10.1590/1808-057 X201602980

Huberty, C. \& Petroskey, M. (2000). Multivariate analysis of variance and covariance. En H. Tinsley y S. Brown (Eds.), Handbook 
Estilos de pensamiento en estudiantes universitarios de Buenos Aires / Freiberg et al.

of applied multivariate statistics and mathematical modeling (pp. 183-208). Nueva York, NY: Academic Press. https://doi.org/ 10.1016/B978-012691360-6/50008-2

Issahaku, P. A. (2017). An Investigation of Factors that Influence the Academic Performance of Undergraduate Students of Public Universities in Ghana. Mediterranean Journal of Social Sciences, 8(3), 259-266. https://doi.org/10.5901/mjss.2017.v8n3p259

Kun, A., Kiss, M. \& Kapitány, A. (2015). The effect of personality on academic performance: Evidence from two university majors. Business Education \& Accreditation, 7(1), 13-24.

Lechner, A. \& Paul, M. (2017). Is this smile for real? The role of affect and thnming styles in customer perceptions of frontline employee emotion authenticity. Journal of Business Research. https://doi.org/10.1016/j.jbusres.2017.06.009

León, F. (2009). Análisis psicométrico del Inventario de Estilo de Pensamiento en una muestra de adolescentes bogotanos escolarizados. Avances en Medición, 7, 103-114.

Maricuţoiu, L. \& Paloş, R. (2014). Adaptation of the Thinking Styles Inventory (TSI) within a Romanian students' sample. Romanian Journal of Applied Psychology, 16(1), 20-24.

Mills, A., Grant, D., Lechner, W. \& Judah, M. (2014). Relationship between trait repetitive negative thinking styles and symptoms of psychopathology. Personality and Individual Differences, 71, 19-24. https://doi.org/10.1016/j.paid.2014.07.022

Siglas (2013). Anuario 2013. Recuperado de http://informacionpresupuestaria.siu.edu.ar/DocumentosSPU/Anuario_2013.pdf

Ministerio de Educación de la Nación Argentina. (2013). Anuario 2013. Estadísticas Universitarias. Recuperado de http://informacionpresupuestaria.siu.edu.ar/DocumentosSPU/Anuario_2013.pdf

Ministerio de Educación y Deportes de la Nación Argentina (2016). Sintesis de información estadísticas universitarias. República Argentina. Recuperado de http://www.educacion.gob.ar/data_storage/file/ documents/sintesis-2016-version-final-ok-58c69d4729ba7.pdf 
Negahi, M., Nouri, N. \& Khoram, A. (2015). The study of learning styles, thinking styles, and english language academic selfefficacy among the students of Islamic Azad University of Behbahan considering their field of study and gender. Theory and Practice in Language Studies, 5(8), 1722-1729. https://doi.org/ 10.17507/tpls.0508.25

Nikčević, A., Marino, C., Caselli, G. \& Spada, M. (2017). The importance of thinking styles in predicting binge eating. Eating Behaviors, 26, 40-44. https://doi.org/10.1016/j.eatbeh. 2017.01.002

Ochiai, J., Maie, Y. \& Wada, Y. (2016). Reliability and validity of the Japanese version of the Thinking Style Inventory. The Japanese Journal of Psychology, 87(2), 172-178. https://doi.org/10.2466/ PR0.92.1.131-140

Organización de las Naciones Unidas para la Educación, la Ciencia y la Cultura (2017). Informe de seguimiento de la educación en el mundo 2016. París: Unesco.

Osborne, J. (2002). Notes on the use of data transformations. Practical Assessment, Research and Evaluation, 8(6), 1-7.

Piaw, C. (2014). Effects of gender and thinking style on student's creative thinking ability. Procedia-Social and Behavioral Sciences, 116, 5135-5139. https://doi.org/10.1016/j.sbspro.2014.01.1087

Pintos, M.E. (2012, 26 de marzo). En el primer año, el 58\% de los estudiantes dejan o cambian de carrera. Clarin.com, Sociedad, Recuperado de http://www.clarin.com/sociedad/primer-estudiantes-dejan-cambian-carrera_0_670732977.html

Planas-Coll, J. \& Enciso-Avila, I. (2014). Los estudiantes que trabajan: ¿tiene valor profesional el trabajo durante los estudios? Revista Iberoamericana de Educación Superior, 12(5), 23-45. https://doi. org/10.1016/S2007-2872(14)71941-9

Pozón-López, J. (2014). Los estudiantes universitarios ante las actividades extracurriculares. Revista Andaluza de Ciencias Sociales, 13, 137-150. https://doi.org/10.12795/anduli.2014.i13.08 
Estilos de pensamiento en estudiantes universitarios de Buenos Aires / Freiberg et al.

Renzulli, S. (2015). Using learning strategies to improve the academic performance of university students on academic probation. NACADA, 35(1), 29-41. https://doi.org/10.12930/ NACADA-13-043

Ross, S. (2007). Introducción a la estadística. Barcelona: Reverté.

Sagone, E. \& De Caroli, M. (2013). Relationships between resilience, self-efficacy, and thinking styles in Italian middle adolescents. Procedia-Social and Behavioral Sciences, 92, 838-845. https:// doi.org/10.1016/j.sbspro.2013.08.763

Shahzad, K., Ahmed, F. \& Ghaffar, A. (2013). Personality and gender as predictors of academic choices: A comparative study of Business and Non-Business students. International Journal of Management \& Organizational Studies, 2(2), 1-7.

Shekhar, C. \& Devi, R. (2012). Achievement motivation across gender and different academic majors. Journal of Educational and Developmental Psychology, 2(2), 105-109. https://doi. org/10.5539/jedp.v2n2p105

Solans, N. \& Lichtmann, T. (2016). Influencias de las nuevas tecnologías de información y comunicación en niños y jóvenes del siglo XXI. Psocial, 2(1), 16-26.

Stanley, V., Bansh, G. \& Om, P. (2011). Thinking styles and academic performance among tribal and non-tribal students. Psychological Studies, 56(2), 220-231. https://doi.org/10.1007/ s12646-011-0076-z

Sternberg, R. (1997). Thinking styles. Nueva York, NY: Cambridge University Press. https://doi.org/10.1017/CBO9780511584152

Sternberg, R. \& Williams, W. (2009). Educational Psychology. Boston: Pearson.

Sternberg, R. \& Zhang, L. (2010). Styles of thinking as a basis of differentiated instruction. Theory into Practice, 44(3), 245-253. https://doi.org/10.1207/s15430421tip4403_9

Strauss, W. \& Howe, N. (1991). Generations: The history of America's future, 1584 to 2069. Nueva York, NY: Harper Perennial. 
Tabachnick, B. \& Fidell, L. (2013). Using multivariate statistics. Boston: Pearson.

Tejedor-Tejedor, F.J. (2003). Poder explicativo de algunos determinantes del rendimiento académico en los estudios universitarios. Revista Española de Pedagogía, 224, 5-32.

Teshome, W. (2018). Psychological factors affecting students' academic performance among freshman psychology students in Dire Dawa University. Journal of Education and Practice, 9(4), 59-65.

Tulbure, C. (2012). Learning styles, teaching strategies and academic achievement in higher education: A cross-sectional investigation. Procedia-Social and Behavioral Sciences, 33, 398-402. https:// doi.org/10.1016/j.sbspro.2012.01.151

Tung, S. \& DeSa, C. (2010). A study of learning and thinking style preferences among urban and rural children. Indian Journal of Community Psychology, 6(2), 168-180.

Turki, J. (2012). Thinking styles "In light of Sternberg's theory" prevailing among the students of Tafila technical University and its relationship with some variables. American International Journal of Contemporary Research, 2(3), 140-152.

Udoh, N. \& Sanni, K. (2012). Parental background variables and the career choice of secondary school students in Uyo local government area, Nigeria. Mediterranean Journal of Social Sciences, 3(1), 497-504.

Wang, L., Wang, S., Keller, L. \& Li, J. (2016). Thinking styles affect reactions to brand crisis apologies. European Journal of Marketing, 50(7), 1263-1289. https://doi.org/10.1108/EJM-07-2014-0457

Wilson, A., Farkas, K., Ishler, K., Gearhart, M., Morgan, R. \& Ashe, M. (2014). Criminal thinking styles among people with serious mental illness in jail. Law and Human Behavior, 38(6), 692-601. https://doi.org/10.1037/lhb0000084

Yu, T. \& Chen, C. (2012). Thinking styles and preferred teacher interpersonal behavior among Hong Kong students. Learning and Individual Differences, 22(4), 554-559. https://doi.org/ $10.1016 /$ j.lindif.2012.02.002 
Yuan, W., Zhang, L. \& Fu, M. (2017). Thinking styles and academic stress coping among Chinese secondary school students. Educational Psychology, 37(8), 1015-1025. https://doi.org/10.1 080/01443410.2017.1287343

Yun-Dai, D. \& Feldhusen, J. (1999). A validation study of the Thinking Styles Inventory: implications for gifted education. Roeper Review, 21(4), 302-307. https://doi.org/10.1080/02783199909553981

Zare, B. (2011). Creativity differences between Art and Engineering Students. International Conference on E-Business, Management and Economics, 25, 207-211.

Zeigler-Hill, V., Mandracchia, J., Dahlen, E., Shango, R. \& Vrabel, J. (2017). Pathological personality traits and criminogenic thinking styles. Personality and Individual Differences, 110, 41-48. https://doi.org/10.1016/j.paid.2017.01.021

Zhang, L. (2004). Revisiting the predictive power of thinking styles for academic performance. The Journal of Psychology, 138(4), 351-370. https://doi.org/10.3200/JRLP.138.4.351-370

Zhang, L. (2010). Further investigating thinking styles and psychosocial development in the Chinese higher education context. Learning and individual Differences, 20, 593-603. https://doi.org/10.1016/ j.lindif.2010.04.011

Zhang, L. \& Sternberg, R. (2005). A threefold model of intellectual styles. EducationalPsychology Review, 17(1), 1-53. https://doi.org/ 10.1007/s10648-005-1635-4

Zhang, L. \& Sternberg, R. (2006). The nature of intelectual styles. Mahwah, NJ: LEA.

Recibido: 26 de octubre, 2018

Revisado: 31 de julio, 2019 Aceptado: 13 de agosto, 2019 